OPEN ACCESS

Edited by:

Feng Gao,

Tianjin University, China

Reviewed by:

Tim Cooper,

University of Houston, USA

Franz Allerberger,

Austrian Agency for Health and Food

Safety, Austria

${ }^{*}$ Correspondence: Alexander W. Friedrich

alex.friedrich@umcg.n

${ }^{\dagger}$ These authors have contributed equally to this work.

Specialty section: This article was submitted to

Evolutionary and Genomic

Microbiology,

a section of the journal

Frontiers in Microbiology

Received: 21 July 2016 Accepted: 14 September 2016 Published: 28 September 2016

Citation:

Ferdous M, Kooistra-Smid AMD,

Zhou K, Rossen JWA and

Friedrich AW (2016) Virulence, Antimicrobial Resistance Properties and Phylogenetic Background of Non-H7 Enteropathogenic Escherichia coli O157. Front. Microbiol. 7:1540. doi: 10.3389/fmicb.2016.01540

\section{Virulence, Antimicrobial Resistance Properties and Phylogenetic Background of Non-H7 Enteropathogenic Escherichia coli 0157}

\author{
Mithila Ferdous ${ }^{1}$, Anna M. D. Kooistra-Smid ${ }^{1,2}$, Kai Zhou ${ }^{1,3}$, John W. A. Rossen ${ }^{1 \dagger}$ and \\ Alexander W. Friedrich ${ }^{1 * \dagger}$
}

\begin{abstract}
${ }^{1}$ Department of Medical Microbiology, University Medical Center Groningen, University of Groningen, Groningen, Netherlands, ${ }^{2}$ Department of Medical Microbiology, Certe Laboratory for Infectious Diseases, Groningen, Netherlands, ${ }^{3}$ State Key Laboratory for Diagnosis and Treatment of Infectious Diseases, Collaborative Innovation Centre for Diagnosis and Treatment of Infectious Diseases, The First Affiliated Hospital, School of Medicine, Zhejiang University, Hangzhou, China
\end{abstract}

Escherichia coli (E.coli) O157 that do not produce Shiga toxin and do not possess flagellar antigen $\mathrm{H} 7$ are of diverse $\mathrm{H}$ serotypes. In this study, the antibiotic resistance properties, genotype of a set of virulence associated genes and the phylogenetic background of E. coli O157:non-H7 groups were compared. Whole genome sequencing was performed on fourteen O157:non-H7 isolates collected in the STEC-ID-net study. The genomes were compared with E. coli $\mathrm{O} 157$ genomes and a typical Enteropathogenic E. coli (tEPEC) genome downloaded from NCBI. Twenty-six (86\%) of the analyzed genomes had the intimin encoding gene eae but of different types mostly correlating with their $\mathrm{H}$ types, e.g., H16, H26, H39, and $\mathrm{H} 45$ carried intimin type $\varepsilon, \beta, \kappa$, and $\alpha$, respectively. They belonged to several $E$. coli phylogenetic groups, i.e., to phylogenetic group A, B1, B2, and D. Seven (50\%) of our collected O157:non-H7 isolates were resistant to two or more antibiotics. Several mobile genetic elements, such as plasmids, insertion elements, and pathogenicity islands, carrying a set of virulence and resistance genes were found in the E. coli O157:non- $\mathrm{H} 7$ isolates. Core genome phylogenetic analysis showed that O157:non-H7 isolates probably evolved from different phylogenetic lineages and were distantly related to the $E$. coli $\mathrm{O} 157: \mathrm{H} 7$ lineage. We hypothesize that independent acquisition of mobile genetic elements by isolates of different lineages have contributed to the different molecular features of the $\mathrm{O} 157$ :non-H7 strains. Although distantly related to the STEC O157, E. coli O157:non-H7 isolates from multiple genetic background could be considered as pathogen of concern for their diverse virulence and antibiotic resistance properties.

Keywords: enteropathogenic Escherichia coli (EPEC), antimicrobial resistance, virulence, mobile genetic elements, whole genome sequencing, phylogenetic relationship, evolution 


\section{INTRODUCTION}

Enteropathogenic Escherichia coli (EPEC) was first identified in the United Kingdom in the 1940s as the cause of outbreaks of infantile diarrhea (Bray, 1945). Although such outbreaks are now rare in developed countries, EPEC strains continue to be a leading cause of diarrhea among infants from developing countries (Chen and Frankel, 2005). The most important feature of EPEC pathogenesis is its ability to produce characteristic histopathological intestinal lesions known as "attaching and effacing" (A/E) lesions that are also characteristic for some Shiga toxin-producing E. coli (STEC) (Jerse et al., 1990). The genes responsible for this activity in EPEC are encoded on a $35-\mathrm{kb}$ pathogenicity island (PAI) called the locus of enterocyte effacement (LEE), whereas the LEE of STEC contains some additional genes encoded within a putative prophage designated 933L (Perna et al., 1998). Both EPEC and STEC LEE encode a type III secretion system, multiple secreted proteins, and a bacterial adhesin called intimin (Nataro and Kaper, 1998). The $5^{\prime}$ regions of intimin-encoding gene eae are conserved in both EPEC and STEC, whereas the $3^{\prime}$ regions are heterogeneous even within the same pathotype (Blanco et al., 2004). Several variants of the eae gene encoding different intimin types and subtypes are described (Oswald et al., 2000; Zhang et al., 2002; Blanco et al., 2006) and it has been suggested that different intimins may be responsible for different host tissue cell tropisms (Torres et al., 2005).

EPEC, carrying EPEC adherence factor plasmid (pEAF) is named as typical EPEC (tEPEC), while those without the pEAF as atypical EPEC (aEPEC) (Trabulsi et al., 2002; Hernandes et al., 2009). aEPEC are thought to be a concern as they can acquire Shiga toxin (Stx) converting bacteriophages, thereby obtaining the ability to cause more serious illness (Bolton et al., 2014). Besides the virulence genes encoded on LEE, EPEC strains also carry other virulence genes including ast $A$ (heat stable enterotoxin), the $c d t$ (cytolethal distending toxin) gene cluster, efa 1 (enterohemorrhagic E. coli factor for adherence), and paa (porcine attaching-effacing associated protein) (Bouzari and Varghese, 1990; Badea et al., 2003; Batisson et al., 2003; Dulguer et al., 2003).

Antibiotic resistance is a global concern due to the increased use of antibiotics, especially for the intestinal organisms as the gut is a heavily populated niche and resistance genes can be transmitted horizontally via these resistant organisms in the gut (Scaletsky et al., 2010). High prevalence of antimicrobial resistance among EPEC strains has been reported in different countries but the genetic basis for this resistance and the evolutionary consequences are rarely studied (Senerwa et al., 1991; Abe et al., 2009; Medina et al., 2011).

The serotyping of $\mathrm{O}$ antigens (together with the $\mathrm{H}$-flagellar antigen) is used as an effective method to identify various pathogenic clones (Iguchi et al., 2011). The serotype O157 is a predominant serotype of the documented STEC related outbreaks worldwide (Nataro and Kaper, 1998; Caprioli et al., 2005) and is frequently associated with the H7 antigen (encoded by $\mathrm{fliC}$ H7). E. coli O157:H7 strains that do not possess Stx are presumably the Stx-lost variants or progenitors of STEC as they share the same phylogenetic lineage (Ferdous et al., 2015). However, the large and diverse O157 serogroup also includes many non-H7 serotypes that are commonly found in animals, food, and clinical samples. EPEC strains of the O157:H45 serotype have been noted as agents of diarrhea in outbreaks and in sporadic cases in Germany, Japan, Korea, and Thailand (Blank et al., 2003; Park et al., 2014). Strains of serotype O157:H8 and O157:H16 have been isolated from cases of diarrhea in human, whereas the latter serotype was also isolated from cattle, beef, and water (Feng et al., 2010; Iguchi et al., 2011). As these strains do not carry the stx gene, they are often not detected or no detailed characterization is carried out (Feng et al., 2010). Recently, two studies were performed on comparative genomics analysis and phylogenetic relationship of E. coli O157:H7 and non-H7 strains (Sanjar et al., 2015; Kossow et al., 2016). However, there is a lack of information regarding their virulence, antimicrobial resistance properties and some other molecular features. In our current study, apart from the phylogenetic and evolutionary relationship of $E$. coli O157:non-H7 groups, a comprehensive characterization was performed focusing on their diversity in virulence and antimicrobial resistance properties.

\section{MATERIALS AND METHODS}

\section{Selection of Isolates for the Study}

A total of 14 E. coli $\mathrm{O} 157$ strains that were negative for $\mathrm{fliC}$ H7 gene were selected for this study. Isolates were obtained from fecal samples of patients with gastrointestinal complaints in the regions of Groningen and Rotterdam during the period April 2013-March 2014, as part of a large multicenter study (STEC-ID-net, unpublished data).

Additionally, publically available genomes of $16 \mathrm{E}$. coli including two O157:H7, one O55:H7, 12 O157:non-H7 and one tEPEC strain (E2348/69; O127:H6) were included in the comparative analyses. The information of isolates and downloaded genomes used in this study are presented in Table 1.

\section{Phenotypic Characterization and Antibiotic Resistance Profile}

Sorbitol fermentation was determined using CT-SMAC plates (sorbitol MacConkey agar with cefixime and tellurite, Mediaproducts BV, Groningen, the Netherlands). Motility was tested using Motility test medium with triphenyltetrazolium chloride (Mediaproducts BV, Groningen, the Netherlands). The production of beta-glucuronidase and urease were checked by using MacConkey II agar with 4-methylumbelliferryl- $\beta$-Dglucuronide (MUG) (BD Diagnostics, Breda, the Netherlands) and urea-triple sugar iron (TSI) agar (Mediaproducts BV, Groningen, the Netherlands), respectively. The $\mathrm{O}$ and $\mathrm{H}$ serotypes of the isolates were determined by seroagglutination performed at the National Institute for Public Health and the Environment (RIVM, Bilthoven, the Netherlands). Antibiotic resistance patterns of the isolates were determined using VITEK2 (bioMérieux, Marcy l'Etoile, France) following EUCAST guidelines. 
TABLE 1 | $E$. coli isolates used in this study.

\begin{tabular}{|c|c|c|c|c|c|c|c|c|c|}
\hline Isolate ID & Isolation regiona & Source & Symptoms ${ }^{b}$ & Genoserotype & MLST & $\begin{array}{l}\text { Phylogenetic } \\
\text { group }\end{array}$ & $\begin{array}{l}\text { Intimin } \\
\text { type }\end{array}$ & Accession No. & References \\
\hline EPEC 400 & Groningen (NL) & Human & $\mathrm{D}$ & O157:H16 & 10 & $A$ & $\varepsilon$ & LZDU00000000 & This study \\
\hline EPEC 536 & Groningen (NL) & Human & ND & O157:H16 & 10 & $A$ & $\varepsilon$ & LZDV00000000 & This study \\
\hline EPEC 631 & Groningen (NL) & Human & $N$ & O157:H16 & 10 & A & $\varepsilon$ & LZDW00000000 & This study \\
\hline EPEC 720 & Groningen (NL) & Human & $N$ & O157:H16 & 10 & A & $\varepsilon$ & LZDX00000000 & This study \\
\hline EPEC 1316 & Groningen (NL) & Human & $\mathrm{D}$ & O157:H16 & 10 & $A$ & $\varepsilon$ & LZDY00000000 & This study \\
\hline EPEC 2646 & Rotterdam (NL) & Human & $N$ & O157:H16 & 10 & $A$ & $\varepsilon$ & LZDZ00000000 & This study \\
\hline EPEC 2669 & Rotterdam (NL) & Human & $N$ & O157:H16 & 10 & A & $\varepsilon$ & LZEA00000000 & This study \\
\hline EPEC 3029 & Rotterdam (NL) & Human & $N$ & O157:H16 & 10 & A & $\varepsilon$ & LZEB00000000 & This study \\
\hline EPEC 1150 & Groningen (NL) & Human & $\mathrm{D}$ & O157:H39 & 4554 & B2 & $\kappa$ & LZEC00000000 & This study \\
\hline EPEC 1554 & Groningen (NL) & Human & $\mathrm{D}$ & O157:H39 & 4554 & B2 & $\kappa$ & LZED00000000 & This study \\
\hline EPEC 2252 & Rotterdam (NL) & Human & $\mathrm{D}$ & O157:H39 & 4554 & B2 & $\kappa$ & LZEE00000000 & This study \\
\hline EPEC 2272 & Rotterdam (NL) & Human & $\mathrm{D}$ & O157:H39 & 4554 & B2 & $\kappa$ & LZEF00000000 & This study \\
\hline EPEC 2081 & Rotterdam (NL) & Human & $N$ & O157:H26 & 189 & $A$ & $\beta$ & LZEG00000000 & This study \\
\hline EPEC 2827 & Rotterdam (NL) & Human & $\mathrm{D}$ & O157:H26 & 189 & $A$ & $\beta$ & LZEH00000000 & This study \\
\hline Sakai & Japan & Human & HUS & O157:H7 & 11 & $\mathrm{D}$ & $\gamma$ & NC_002695 & Hayashi et al., 2001 \\
\hline SS52 & USA & Cattle & NA & O157:H7 & 11 & $\mathrm{D}$ & $\gamma$ & СР010304 & Katani et al., 2015 \\
\hline CB9615 & Germany & Human & $\mathrm{D}$ & O55:H7 & 335 & $\mathrm{D}$ & $\gamma$ & CP001846 & Zhou et al., 2010 \\
\hline Santai & China & Duck & NA & O157:H16 & 1011 & $\mathrm{D}$ & NA & СР007592 & Cheng et al., unpublished \\
\hline 3006 & USA & Human & $N$ & O157:H16 & 5502 & A & NA & AMUN01000000 & Sanjar et al., 2015 \\
\hline TW15901 & France & Food & NA & O157:H16 & 10 & A & $\varepsilon$ & AMUK01000000 & Sanjar et al., 2015 \\
\hline TW00353 & USA & Human & $N$ & O157:H16 & 10 & $A$ & $\varepsilon$ & AMUM01000000 & Sanjar et al., 2015 \\
\hline C639_08 & Denmark & Human & $N$ & O157:H45 & 725 & B2 & $\alpha$ & AlBH00000000 & Hazen et al., 2013 \\
\hline C844_97 & Japan & Human & $N$ & O157:H45 & 725 & B2 & $\alpha$ & AlBZ01000000 & Hazen et al., 2013 \\
\hline RN587/1 & Brazil & Human & $N$ & O157:H45 & 725 & B2 & $\alpha$ & ADUS01000000 & Hazen et al., 2013 \\
\hline ARS4.2123 & USA & Water & NA & O157:H45 & 725 & B2 & $\alpha$ & AMUL01000000 & Sanjar et al., 2015 \\
\hline TW07793 & Unknown & Water & NA & O157:H39 & 1041 & B2 & $\kappa$ & AFAG0200000 & Sanka et al., unpublished \\
\hline 7798 & Argentina & Human & $N$ & O157:H39 & 5611 & B2 & $\kappa$ & AMUP00000000 & Sanjar et al., 2015 \\
\hline N1 & Unknown & Food & NA & O157:H29 & 515 & B1 & NA & AMUQ01000000 & Sanjar et al., 2015 \\
\hline T22 & Hungary & Human & $N$ & O157:H43 & 155 & B2 & NA & AHZD02000000 & Sanjar et al., 2015 \\
\hline E2348/69 & Taunton, United Kingdom & Human & $\mathrm{D}$ & O127:H6 & 15 & B2 & $\alpha$ & FM180568 & Iguchi et al., 2009 \\
\hline
\end{tabular}

${ }^{a} N L$, the Netherlands.

${ }^{b} D$, Diarrhea; ND, Abdominal pain and other gastrointestinal problems without diarrhea; N, Not available; HUS, Hemolytic uremic syndrome; NA, Not applicable.

\section{Whole Genome Sequencing}

From all the isolates DNA was extracted using the UltraClean ${ }^{\circledR}$ microbial DNA isolation kit (MO BIO Laboratories, Carlsbad, CA, US) according to the manufacturer's protocol. A DNA library was prepared using the Nextera XT kit (Illumina, San Diego, CA, US) according to the manufacturer's instructions and then ran on a Miseq (Illumina) for generating paired-end 250-bp reads aiming at a coverage of at least 60 fold as described previously (Ferdous et al., 2015; Zhou et al., 2015).

\section{Data Analysis and Molecular Typing}

De novo assembly was performed using CLC Genomics Workbench v7.0.3 (CLC bio A/S, Aarhus, Denmark) after quality trimming (Qs $\geq 28$ ) with optimal word sizes based on the maximum N50 value (Ferdous et al., 2015; Zhou et al., 2015). All the assembled genomes of this study and the assembled genomes downloaded from NCBI were subjected to further analyses. Annotation was performed by uploading the assembled genomes onto the RAST server version 2.0 (Aziz et al., 2008). The sequence type (ST) was identified by uploading the assembled genomes to the Center for Genomic Epidemiology (CGE) MLST finder website (version 1.7) (Larsen et al., 2012). For four of the isolates the allele numbers were submitted to the E. coli MLST databases (http://mlst.warwick.ac.uk/mlst/dbs/Ecoli) to obtain a ST for them. The virulence genes were determined by virulence finder 1.2 (Joensen et al., 2014), antibiotic resistance genes were determined by ResFinder 2.1 (Zankari et al., 2012) and the serotyping genes of the isolates were confirmed using the SeroTypeFinder tool (Joensen et al., 2015) from the CGE server. Intimin types of the isolates were determined using blastn. Isolates were assigned to one of the major $E$. coli phylogenetic groups A, B1, B2, or D using the genetic markers chuA, yjaA, and the DNA fragment TspE4.C2 (Clermont et al., 2000).

\section{Synteny Analysis of Resistance and Virulence Genes}

To determine the location of virulence and antibiotic resistance genes in the isolates, the contigs containing the targeted genes 
were subjected to BLAST in NCBI to look for the most related genomic regions which were later used as reference to confirm their presence in our analyzed isolates.

To compare the sequences of the LEE region, the LEE of the E. coli O157:H7 strain 71074 (accession no GQ338312) was used as the reference and the contigs of each sample were subjected to BLAST against the reference and plotted by BLAST ring image generator (BRIG) (Alikhan et al., 2011).

\section{Phylogenetic Analysis}

To determine the phylogenetic relationship of the isolates, a geneby-gene approach was performed using SeqSphere ${ }^{+}$v3.0 (Ridom $\mathrm{GmbH}$, Münster, Germany). For this, an ad-hoc core genome MLST (cgMLST) scheme was used as described previously (Ferdous et al., 2016). Briefly, the genome of E. coli O157:H7 strain Sakai was taken as a reference genome and ten additional E. coli genomes were used as query genomes to extract open reading frames (ORFs) using MLST+ Target Definer 2.1.0 of $\mathrm{SeqSphere}^{+}$. Only the ORFs without premature stop codon and ambiguous nucleotides from contigs of assembled genomes were included. The genes shared by the genomes of all isolates analyzed in this study were defined as the core genome for phylogenetic analysis (Ferdous et al., 2016). A Neighbor Joining (NJ) tree was constructed based on a distance matrix among differences in the core genome of the isolates.

\section{RESULTS}

\section{Phenotype}

Ten of the fourteen O157 isolates collected in our study were motile and were of serotype O157:H16 $(n=8)$ or O157:H26 $(n=2)$; the other four were non-motile. All the isolates were positive for beta-glucuronidase and negative for urease. Except two isolates (EPEC 2646 and EPEC 2669) all grew on CT-SMAC agar and fermented sorbitol.

\section{Molecular Typing}

The molecular typing results of the E. coli O157 isolates included in this study are summarized in Table 1. The four non-motile isolates of this study were confirmed to carry the $\mathrm{fliC} \mathrm{H} 39$ gene and were assigned to a new ST (ST 4554). All of the O157:H16 isolates belonged to $E$. coli phylogenetic group A except strain Santai which belonged to phylogenetic group D (phylogenetic group of STEC O157:H7). None of the strains were positive for the stx gene.

\section{Virulence Profiling and Intimin Typing}

The virulence profiles of the isolates and of the genomes are presented in Figure 1. Four of the genomes obtained from NCBI (N1, T22, 3006, and Santai) did not have the eae gene. The rest carried the gene but different types of it were found mostly correlating to their genoserotypes, e.g., O157:H16 and O157:H39 isolates carried intimin type $\varepsilon$ and $\kappa$ respectively (Table 1 ). None of the isolates carried intimin $\gamma$. Like tEPEC E2348/69, all O157:H45 strains had intimin $\alpha$.

Besides the virulence genes located on the LEE PAI, isolates contained other virulence genes carried on several mobile genetic elements (MGEs), e.g., the plasmid-borne genes etpD, toxB, $\operatorname{sep} A$, the prophage encoded genes espI, $t c c P$, cif, the PAI related genes iha and espC, and other virulence determinants carried on insertion elements or transposons (Figure 1). All O157:H39 isolates carried the etpD gene located on pO55 like (O55:H7 strain CB9615) plasmid encoding the type II secretion system proteins (etp gene cluster) and the conjugal transfer proteins (tra gene cluster) (Supplementary Figure S1), and adhesion gene iha presumably on a transposon. All of the O157:H45 and O157:H39 strains carried the espC PAI (Supplementary Figure S2). All the isolates analyzed in this study were negative for the pillin subunit encoding gene $s f p \mathrm{~A}$.

\section{Comparison of LEE}

Figure 2 shows the variations in the sequence of the LEE PAI in EPEC $\mathrm{O} 157$ isolates with different $\mathrm{H}$ types. The first $8 \mathrm{~kb}$ region, encoding the phage associated integrase and IS elements in STEC LEE, is not intact in EPEC isolates and some of them only contain part of it. For example, EPEC O157:H16 and EPEC 2827 isolates contain the insertion element IS66 and O157:H26 isolates contain a similar but not identical phage integrase as present in the STEC LEE. Among the core LEE genes, EPEC secreted proteins encoding genes (esp genes) and the genes encoding the proteins for adhesion (e.g., eae, tir-encoding translocated intimin receptor) of the EPEC isolates are different from those of STEC. Other genes encoding type III secretion system (e.g., esc and sep genes) and the regulator gene ler are similar in O157:H39 and O157:H45 EPEC isolates (represented by dark blue and dark pink color in Figure 2, respectively) to those of STEC but are different in O157:H16 and O157:H26 (represented by the relatively light green and orange colors in Figure 2, respectively). The sequence of the tir gene is conserved between O157:H16 and O157:H26 serotypes (97-98\% similar), and between O157:H39 and O157:H45 serotypes (98-99\% similar). Notably, the tir gene of H39 and H45 is similar (97-98\%) to that of tEPEC E2348/69, whereas in $\mathrm{H} 16$ and $\mathrm{H} 26$ it is different ( $<78 \%$ similarity) from all other serotypes analyzed in this study (data not shown). Clearly, the sequence of the LEE region is variable in different O157 EPEC strains.

\section{Antibiotic Resistance Profile and Presence of Resistance Genes}

Among the fourteen $\mathrm{O} 157$ isolates collected in this study, all O157:H39 and O157:H26 isolates and one of the O157:H16 isolates (EPEC 1316) were resistant to two or more antibiotics and EPEC 2827 was resistant to several antibiotics. The presence of antibiotic resistance genes was determined for all the genomes analyzed in this study (including those downloaded from NCBI without available phenotypic data) and the results are presented in Table 2. More than ten resistance genes were found in two isolates, EPEC 2827 and Santai. EPEC 2827 carried the resistance gene strA on a plasmid similar to pSTU288 (accession no. CP004059) of Salmonella enterica (Figure 3A), and $d f r \mathrm{~A}, \operatorname{aph} \mathrm{A}, \operatorname{aadA}, b l a \mathrm{OXA}$, and $\operatorname{cat} \mathrm{B}$ on an integron (accession no. HQ386835) of Proteus sp. VIITMP5 (Figure 3B). EPEC 1554 carried the tet A gene on a transposon similar to Tn1721 (accession no. X61367) (Figure 3C). The 


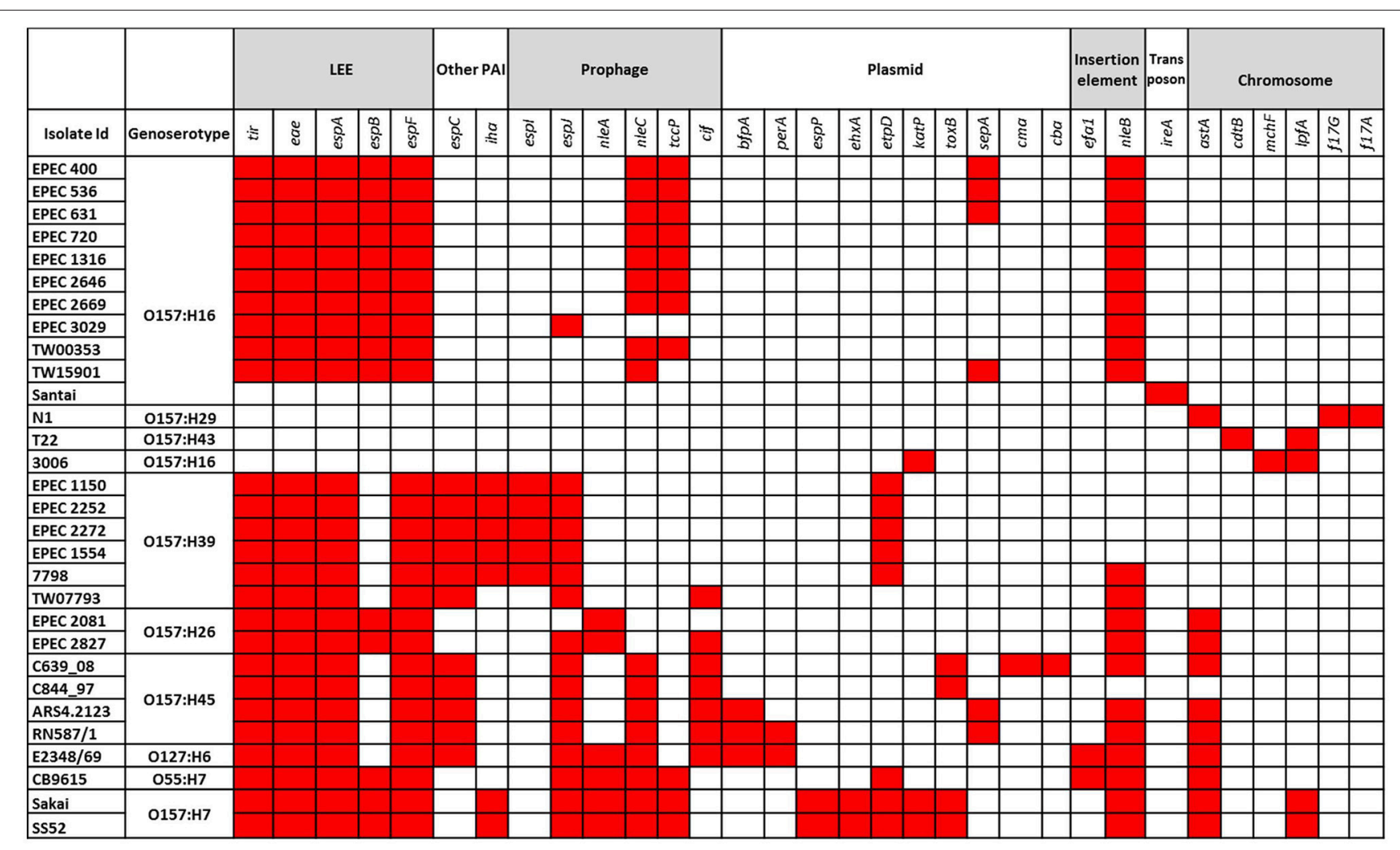

FIGURE 1 | Virulence profiles of the isolates analyzed in this study. Predicted locations of the virulence genes are indicated. The red color indicates the presence of a gene in the corresponding isolate.

O157:H39 strains had a plasmid similar to pCERC1 (accession no. JN012467) of E. coli strain S1.2.T2R carrying a cluster of antibiotic resistance genes including strA-B, sul2, and $d f r A 14$ (Figure 3D). EPEC 1316 carried the strA and blaTEM genes on a plasmid similar to pVR50A (accession no. CP011135) (data not shown) of E. coli VR50 caused an asymptomatic bacteriuria.

\section{Phylogenetic Analysis}

Figure 4 shows a NJ tree based on 2683 genes (core genome). The tree shows that strain Santai (phylogenetic group D) shared a common ancestor with the E. coli O157:H7 lineage and its progenitor O55:H7. The tEPEC O157:H45 (RN587/1 and ARS4.2123) and the aEPEC O157:H45 (C639_08 and C844_97) genomes clustered together and O157:H45 was the serogroup most closely related to tEPEC reference strain E2348/69. O157:H39 isolates were more closely related with tEPEC isolates than the O157:H16 and O157:H26 isolates. Three of the O157:H39 isolates (EPEC 1150, EPEC 2252, and EPEC 2272) appeared to be exactly similar in their core genome. Remarkably, except strain 3006 and Santai, the other ten O157:H16 isolates belonging to ST10 were closely related to each other with allele differences ranging from 0 to 41, whereas Santai and 3006 had a minimum of 2176 allele differences (data not shown) with the other O157:H16 isolates of this study indicating that only one fifth of their core genome contains identical alleles.

\section{DISCUSSION}

E. coli $\mathrm{O} 157$ strains are mostly known as enterohemorrhagic E. coli (EHEC) that have been associated with food born outbreaks worldwide. But they are also comprised of other $E$. coli pathogroups, including ones containing eae and lacking stx, which are considered as EPEC. E. coli O157:H7 isolates lacking stx gene are assumed to be members of STEC that lost the Stx encoding bacteriophages (Ferdous et al., 2015). The current study was performed to compare the molecular features and virulence properties of EPEC $\mathrm{O} 157$ having different $\mathrm{H}$ antigens (other than H7) with those of STEC O157:H7 isolates and to determine their phylogenetic relationship. STEC O157 isolates generally belong to ST11 and carry the $\mathrm{fliC} \mathrm{H} 7$ gene encoding flagellar antigen $\mathrm{H} 7$ but the EPEC O157 isolates appeared to be from different sequence and $\mathrm{H}$ serotypes. The stx negative O157 isolates in this study were usually sorbitol fermenting (SF) and some of them were non-motile. Therefore, they could be misidentified as STEC SF O157:NM if proper molecular tests are not performed (Feng et al., 2010). For some draft genomes obtained from NCBI, prediction of the O157 serotyping gene $w z x$ was difficult probably because of the breaking of contigs in the middle of the gene sequence, therefore a lower threshold of the percentage of minimum overlapping gene length was applied to confirm the genoserotype.

Intimin types of the isolates correlated to their genoserotypes; four of the genomes did not possess intimin and therefore 

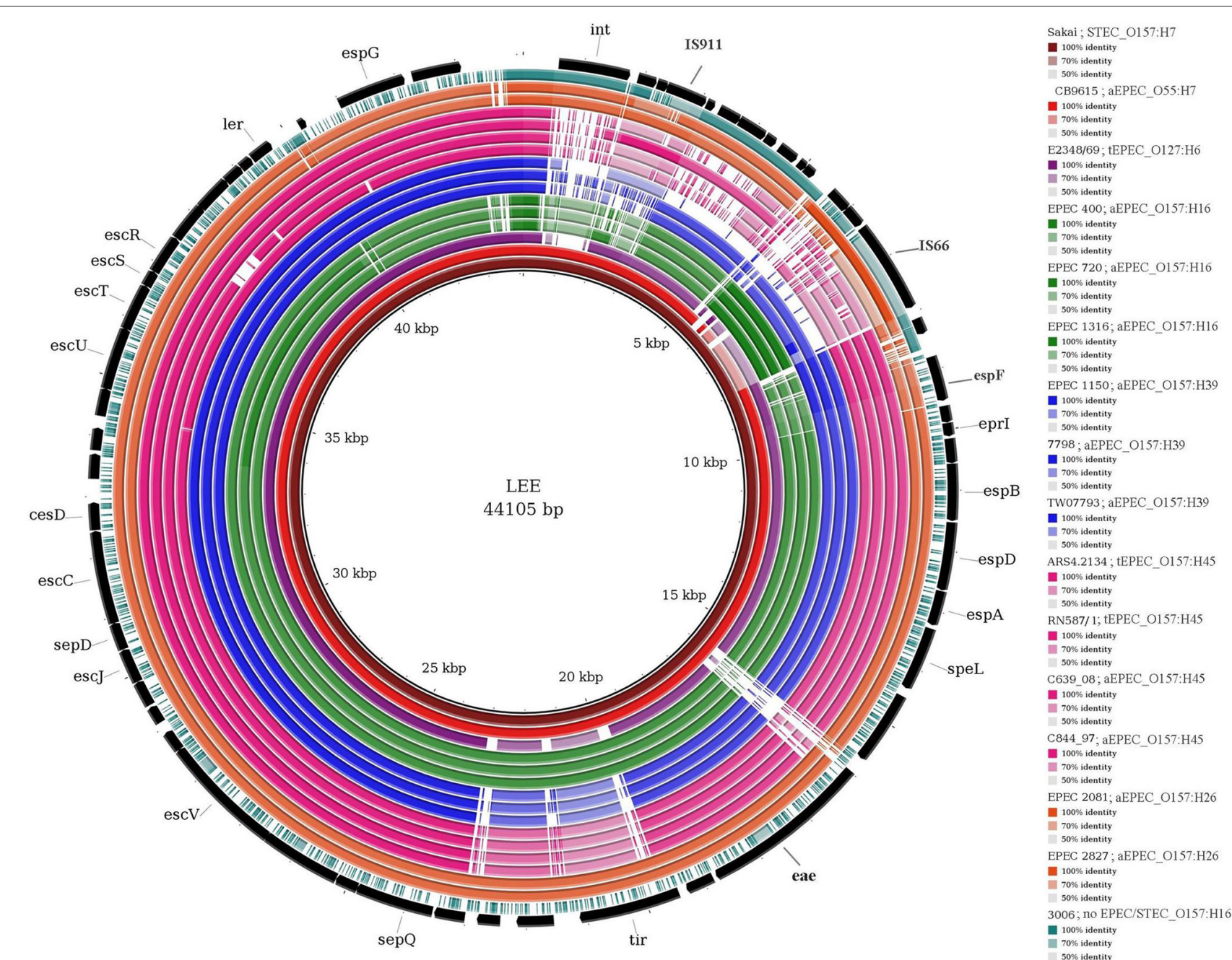

FIGURE 2 | Comparison of LEE in $\boldsymbol{E}$. coli isolates analyzed in this study. The figure shows BLAST comparison of E. coli isolates against the reference LEE sequence (core black circle). Each ring represents one isolate, different colors of the rings represent different genoserotypes. The gradients (dark, pale, and white) of each color represent the sequence similarity (from 100 to $0 \%$ ) between samples and reference. The colors of different isolates as well as the order of the rings (from inner to outer) with the color gradient for sequence identity are shown in the legend (right). Please note that, for each genoserotype only representative isolates showing variations in the LEE sequences are presented in the figure.

were not considered to be EPEC. Our phylogenetic analysis based on cgMLST confirmed that E. coli O157:H7 belong to a different lineage than the non-H7 group, the latter belonging to diverse phylogenetic lineages (Sanjar et al., 2015). Our results support previous findings that horizontal transfer of the $\mathrm{O} 157$ antigen gene cluster has occurred independently among different E. coli lineages (Iguchi et al., 2011). Interestingly, isolates of O157:H45 clustered together independent of being a tEPEC or aEPEC. However, strain C844-97 was previously described to have lost its EAF plasmid (Hazen et al., 2013), and this may also be the case for strain C639-08 explaining their aEPEC features in spite of clustering together with tEPEC strains. Although the whole $b f p$ gene cluster was present in tEPEC strains RN587/1 and ARS4.2123, they did not contain the complete EAF plasmid.
Almost all of the O157:H39 isolates included in this study belonged to recently evolved STs (e.g., ST4554 and ST5611). Alternatively, they were not reported previously, because no further characterization was performed as they did not carry the stx gene. We observed the presence of several plasmids, insertion elements and PAIs in O157:H39 isolates contributing to their virulence and resistance features. Three of the O157:H39 isolates of the new sequence type ST4554, shared an identical core genome but no epidemiological link could be identified among the patients from whom they were isolated.

The distribution of virulence genes almost perfectly correlated to the STs although slight intra-ST variations in virulence patterns were observed. The virulence genes were often carried on plasmids, PAIs, prophages, and insertion elements suggesting that these virulence factors were acquired from numerous 
TABLE 2 | Antibiotic resistance profiles of the analyzed isolates.

\begin{tabular}{|c|c|c|c|}
\hline Isolate ID & Genoserotype & Phenotypical resistance ${ }^{\text {b }}$ & Presence of Resistance genes ${ }^{c}$ \\
\hline EPEC 1316 & O157:H16 & AMP, SXT, TMP & blaTEM-1B, dfrA8, strA/B, sul2 \\
\hline EPEC 1150 & O157:H39 & AMP, SXT, TMP & blaTEM-1B, dfrA14, strA/B, sul2 \\
\hline EPEC 2272 & O157:H39 & AMC, AMP, SXT, TMP & blaTEM-1B, dfrA14, strA/B, sul2 \\
\hline EPEC 1554 & O157:H39 & AMP, TET & blaTEM-1B, tetA \\
\hline C639_08 & $\mathrm{O} 157: \mathrm{H} 45$ & NT & sul2 \\
\hline RN587/1 & O157:H45 & NT & blaTEM-116 \\
\hline Santai & $\mathrm{O} 157: \mathrm{H} 16$ & NT & $\begin{array}{l}\text { aac(3)-Ild, aac( }\left(6^{\prime}\right) \mid l b-c r, \text { aadA, armA, ARR-3, strA/B, } \\
\text { blaOXA-1, blaTEM-1B, catA/B, dfrA12, floR, fosA, } \\
\text { mphA/E, msrE, sul1/2, tetA }\end{array}$ \\
\hline
\end{tabular}

aThe first seven rows shaded gray are isolates collected in our study and their resistance profile was determined using VITEK2.

${ }^{b}$ AMP, ampicillin; AMC, amoxicillin-clavulanic acid; FOX, cefoxitin; CIP, ciprofloxacin; GEN, gentamicin; NIT, nitrofurantoin; NOR, norfloxacin; TET, tetracycline; TOB, tobramycin; TMP, trimethoprim; SXT, trimethoprim-sulfamethoxazole. NT, Not tested as only the genomes of these strains were available.



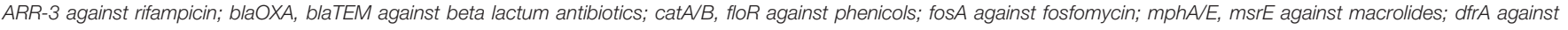
trimethoprim; sul1/2 against sulfonamides; tet against tetracycline.



B VIITMP5 1 (class 1) integron of Proteus sp.

sources via MGE, found specifically in the genome of pathogenic strains (Donnenberg and Whittam, 2001). The lineage specific acquisition of specific combinations of virulence factors may confer selective advantages contributing to the fitness of the pathogens favoring their establishment and transmission as new virulent clones (Reid et al., 2000). It was observed that ten of the O157:H16 isolates of ST10 (including one isolate from the US, one non-human isolate from France, and the rest from the Netherlands) shared almost identical virulence properties and were closely related in cgMLST with a maximum of a 41-allele 




FIGURE 4 | Phylogenetic relationship of the isolates analyzed in this study. This Neighbor Joining tree was constructed based on a distance matrix among differences in the core genome of the isolates. Each isolate ld is followed by its genoserotype, sequence type, and major phylogenetic group.

difference (data not shown). A similar finding was observed in a previous study where O157:H16 strains were described as representatives of a widespread clone (Feng et al., 2010). However, the other two O157:H16 isolates, 3006 and Santai, belonged to two different STs, ST5502, and ST1011, respectively, and possessed almost no virulence genes. They were not classified as EPEC supporting the idea that the $\mathrm{O}$ and $\mathrm{H}$ serotyping might not represent the virulence status of the isolates and that the distribution of $\mathrm{O}$ and $\mathrm{H}$ antigen encoding genes is irrespective of the E. coli pathotype (Blank et al., 2003). Moreover, aEPEC isolates analyzed in this study were distributed in two completely different phylogenetic branches; one containing O157:H39 and O157:H45 (closer related to tEPEC) and another containing O157:H16 and O157:H26. This may be explained by horizontal transfer of the LEE PAI to different E. coli groups contributing to their EPEC feature (Feng et al., 2010).

Strain Santai belonging to the phylogenetic group D as does STEC O157:H7, appears to be more a multidrug resistant strain than a virulent one. The resistance genes were integrated into the chromosome of this organism through insertion elements and transposons from different species. EPEC 2827 was also resistant to several antibiotics and carried the drug resistance genes on a plasmid very similar to that of S. enterica (Hooton et al., 2014) and on an insertion element similar to a class1 integron of Proteus sp. (Guo et al., 2011). Isolates of ST4554 carried a plasmid similar to pCER1, a small, globally disseminated plasmid harboring sulphonamide and streptomycin resistance genes (Anantham and Hall, 2012). EPEC 1316 carried the resistance genes on a plasmid similar to pVR50A which also carries a tra gene cluster encoding conjugal transfer proteins (Beatson et al., 2015). Clearly, the transmission of MGEs plays a role in the evolution of new MDR lineages (Beceiro et al., 2013; Punina et al., 2015). EPEC infections can normally be recovered without antimicrobial therapy, therefore, the persistence of resistant EPEC strains is more likely to be related to selective pressure from antimicrobials applied at the population level or present in the environment (Scaletsky et al., 2010). Indeed the presence of resistance genes in $E$ coli that originated from the environment have been described before (Hamelin et al., 2007).

In conclusion, E. coli O157:non-H7 isolates have evolved from multiple genetic backgrounds. They are distantly related to the most virulent EHEC clonal lineage EHEC O157:H7. Diversity in virulence and antibiotic resistance patterns have been observed among these O157:non-H7 isolates and mobile elements carried by these organisms could play a role in the evolution and dissemination of these virulent and resistant clones. 


\section{AUTHOR CONTRIBUTIONS}

Study design: MF, JR, AF. Performing Experiments: MF. Acquisition of data: MF, AK, JR. Analysis and interpretation of data: MF, KZ, JR, and AF. Drafting of manuscript: MF, KZ, AK, JR, and AF. Critical revision: MF, KZ, AK, JR, and AF.

\section{FUNDING}

This study was partly supported by the Interreg IVa-funded projects EurSafety Heath-net (III-1-02 = 73) and SafeGuard $($ III-2-03 $=025)$.

\section{REFERENCES}

Abe, C. M., Trabulsi, L. R., Blanco, J., Blanco, M., Dahbi, G., Blanco, J. E., et al. (2009). Virulence features of atypical enteropathogenic Escherichia coli identified by the eae (+) EAF-negative stx(-) genetic profile. Diagn. Microbiol. Infect. Dis. 64, 357-365. doi: 10.1016/j.diagmicrobio.2009.03.025

Alikhan, N. F., Petty, N. K., Ben Zakour, N. L., and Beatson, S. A. (2011). BLAST Ring Image Generator (BRIG): simple prokaryote genome comparisons. BMC Genomics 12:402. doi: 10.1186/1471-2164-12-402

Anantham, S., and Hall, R. M. (2012). pCERC1, a small, globally disseminated plasmid carrying the dfrA14 cassette in the strA gene of the sul2-strA-strB gene cluster. Microb. Drug Resist. 18, 364-371. doi: 10.1089/mdr.2012.0008

Aziz, R. K., Bartels, D., Best, A. A., DeJongh, M., Disz, T., Edwards, R. A., et al. (2008). The RAST Server: rapid annotations using subsystems technology. BMC Genomics 9:75. doi: 10.1186/1471-2164-9-75

Badea, L., Doughty, S., Nicholls, L., Sloan, J., Robins-Browne, R. M., and Hartland, E. L. (2003). Contribution of Efa1/LifA to the adherence of enteropathogenic Escherichia coli to epithelial cells. Microb. Pathog. 34, 205-215. doi: 10.1016/S0882-4010(03)00026-3

Batisson, I., Guimond, M. P., Girard, F., An, H., Zhu, C., Oswald, E., et al. (2003). Characterization of the novel factor paa involved in the early steps of the adhesion mechanism of attaching and effacing Escherichia coli. Infect. Immun. 71, 4516-4525. doi: 10.1128/IAI.71.8.4516-4525.2003

Beatson, S. A., Ben Zakour, N. L., Totsika, M., Forde, B. M., Watts, R. E., Mabbett, A. N., et al. (2015). Molecular analysis of asymptomatic bacteriuria Escherichia coli strain VR50 reveals adaptation to the urinary tract by gene acquisition. Infect. Immun. 83, 1749-1764. doi: 10.1128/IAI.02810-14

Beceiro, A., Tomás, M., and Bou, G. (2013). Antimicrobial resistance and virulence: a successful or deleterious association in the bacterial world? Clin. Microbiol. Rev. 26, 185-230. doi: 10.1128/CMR.00059-12

Blanco, M., Blanco, J. E., Blanco, J., de Carvalho, V. M., Onuma, D. L., and Pestana de Castro, A. F. (2004). Typing of intimin (eae) genes in attaching and effacing Escherichia coli strains from monkeys. J. Clin. Microbiol. 42, 1382-1383. doi: 10.1128/JCM.42.3.1382-1383.2004

Blanco, M., Blanco, J. E., Dahbi, G., Mora, A., Alonso, M. P., Varela, G., et al. (2006). Typing of intimin (eae) genes from enteropathogenic Escherichia coli (EPEC) isolated from children with diarrhoea in Montevideo, Uruguay: identification of two novel intimin variants (muB and xiR/beta2B). J. Med. Microbiol. 55, 1165-1174. doi: 10.1099/jmm.0.46518-0

Blank, T. E., Lacher, D. W., Scaletsky, I. C., Zhong, H., Whittam, T. S., and Donnenberg, M. S. (2003). Enteropathogenic Escherichia coli O157 strains from Brazil. Emerg. Infect. Dis. 9, 113-115. doi: 10.3201/eid0901.020072

Bolton, D. J., Ennis, C., and McDowell, D. (2014). Occurrence, virulence genes and antibiotic resistance of enteropathogenic Escherichia coli (EPEC) from twelve bovine farms in the north-east of Ireland. Zoonoses Public Health 61, 149-156 doi: $10.1111 /$ zph.12058

Bouzari, S., and Varghese, A. (1990). Cytolethal distending toxin (CLDT) production by enteropathogenic Escherichia coli (EPEC). FEMS Microbiol. Lett. 59, 193-198. doi: 10.1111/j.1574-6968.1990.tb03821.x

\section{ACKNOWLEDGMENTS}

We would like to thank the project members of the STEC-ID-net study, technicians for performing the whole genome sequencing and Sigrid Rosema for helping in data analysis.

\section{SUPPLEMENTARY MATERIAL}

The Supplementary Material for this article can be found online at: http://journal.frontiersin.org/article/10.3389/fmicb. 2016.01540

Bray, J. (1945). Isolation of antigenically homogenous strains of Bact. coli Neapolitanum from summer diarrhoea of infants. J. Pathol. Bacteriol. 57, 239-247. doi: 10.1002/path.1700570210

Caprioli, A., Morabito, S., Brugère, H., and Oswald, E. (2005). Enterohaemorrhagic Escherichia coli: emerging issues on virulence and modes of transmission. Vet. Res. 36, 289-311. doi: 10.1051/vetres:2005002

Chen, H. D., and Frankel, G. (2005). Enteropathogenic Escherichia coli: unravelling pathogenesis. FEMS Microbiol. Rev. 29, 83-98. doi: 10.1016/j.femsre.2004.07.002

Clermont, O., Bonacorsi, S., and Bingen, E. (2000). Rapid and simple determination of the Escherichia coli phylogenetic group. Appl. Environ. Microbiol. 66, 4555-4558. doi: 10.1128/AEM.66.10.4555-4558.2000

Donnenberg, M. S., and Whittam, T. S. (2001). Pathogenesis and evolution of virulence in enteropathogenic and enterohemorrhagic Escherichia coli. J. Clin. Invest. 107, 539-548. doi: 10.1172/JCI12404

Dulguer, M. V., Fabbricotti, S. H., Bando, S. Y., Moreira-Filho, C. A., FagundesNeto, U., and Scaletsky, I. C. (2003). Atypical enteropathogenic Escherichia coli strains: phenotypic and genetic profiling reveals a strong association between enteroaggregative E. coli heat-stable enterotoxin and diarrhea. J. Infect. Dis. 188, 1685-1694. doi: 10.1086/379666

Feng, P. C., Keys, C., Lacher, D., Monday, S. R., Shelton, D., Rozand, C., et al. (2010). Prevalence, characterization and clonal analysis of Escherichia coli O157: non-H7 serotypes that carry eae alleles. FEMS Microbiol. Lett. 308, 62-67. doi: 10.1111/j.1574-6968.2010.01990.x

Ferdous, M., Friedrich, A. W., Grundmann, H., de Boer, R. F., Croughs, P. D., Islam, M. A., et al. (2016). Molecular characterization and phylogeny of Shiga toxin-producing Escherichia coli isolates obtained from two Dutch regions using whole genome sequencing. Clin. Microbiol. Infect. 22, 642.e1-642.e9. doi: 10.1016/j.cmi.2016.03.028

Ferdous, M., Zhou, K., Mellmann, A., Morabito, S., Croughs, P. D., de Boer, R. F., et al. (2015). Is shiga toxin-negative Escherichia coli O157:H7 enteropathogenic or enterohemorrhagic Escherichia coli? comprehensive molecular analysis using whole-genome sequencing. J. Clin. Microbiol. 53, 3530-3538. doi: 10.1128/JCM.01899-15

Guo, X., Xia, R., Han, N., and Xu, H. (2011). Genetic diversity analyses of class 1 integrons and their associated antimicrobial resistance genes in Enterobacteriaceae strains recovered from aquatic habitats in China. Lett. Appl. Microbiol. 52, 667-675. doi: 10.1111/j.1472-765X.2011.03059.x

Hamelin, K., Bruant, G., El-Shaarawi, A., Hill, S., Edge, T. A., Fairbrother, J., et al. (2007). Occurrence of virulence and antimicrobial resistance genes in Escherichia coli isolates from different aquatic ecosystems within the St. Clair River and Detroit River areas. Appl. Environ. Microbiol. 73, 477-484. doi: 10.1128/AEM.01445-06

Hayashi, T., Makino, K., Ohnishi, M., Kurokawa, K., Ishii, K., Yokoyama, K., et al. (2001). Complete genome sequence of enterohemorrhagic Escherichia coli O157:H7 and genomic comparison with a laboratory strain K-12. DNA Res. 8, 11-22. doi: 10.1093/dnares/8.1.11

Hazen, T. H., Sahl, J. W., Fraser, C. M., Donnenberg, M. S., Scheutz, F., and Rasko, D. A. (2013). Draft genome sequences of three O157 
enteropathogenic Escherichia coli isolates. Genome Announc. 1:e00516-13. doi: 10.1128/genomeA.00516-13

Hernandes, R. T., Elias, W. P., Vieira, M. A., and Gomes, T. A. (2009). An overview of atypical enteropathogenic Escherichia coli. FEMS Microbiol. Lett. 297, 137-149 doi: 10.1111/j.1574-6968.2009.01664.x

Hooton, S. P., Timms, A. R., Cummings, N. J., Moreton, J., Wilson, R., and Connerton, I. F. (2014). The complete plasmid sequences of Salmonella enterica serovar Typhimurium U288. Plasmid 76C, 32-39. doi: 10.1016/j.plasmid.2014.08.002

Iguchi, A., Shirai, H., Seto, K., Ooka, T., Ogura, Y., Hayashi, T., et al. (2011). Wide distribution of O157-antigen biosynthesis gene clusters in Escherichia coli. PLoS ONE 6:e23250. doi: 10.1371/journal.pone.0023250

Iguchi, A., Thomson, N. R., Ogura, Y., Saunders, D., Ooka, T., Henderson, I. R., et al. (2009). Complete genome sequence and comparative genome analysis of enteropathogenic Escherichia coli O127:H6 strain E2348/69. J. Bacteriol. 191, 347-354. doi: 10.1128/JB.01238-08

Jerse, A. E., Yu, J., Tall, B. D., and Kaper, J. B. (1990). A genetic locus of enteropathogenic Escherichia coli necessary for the production of attaching and effacing lesions on tissue culture cells. Proc. Natl. Acad. Sci. U.S.A. 87, 7839-7843. doi: 10.1073/pnas.87.20.7839

Joensen, K. G., Scheutz, F., Lund, O., Hasman, H., Kaas, R. S., Nielsen, E. M., et al. (2014). Real-time whole-genome sequencing for routine typing, surveillance, and outbreak detection of verotoxigenic Escherichia coli. J. Clin. Microbiol. 52, 1501-1510. doi: 10.1128/JCM.03617-13

Joensen, K. G., Tetzschner, A. M., Iguchi, A., Aarestrup, F. M., and Scheutz, F. (2015). Rapid and easy in silico serotyping of Escherichia coli isolates by use of whole-genome sequencing data. J. Clin. Microbiol. 53, 2410-2426. doi: 10.1128/JCM.00008-15

Katani, R., Cote, R., Raygoza Garay, J. A., Li, L., Arthur, T. M., DebRoy, C., et al. (2015). Complete genome sequence of SS52, a strain of Escherichia coli O157:H7 recovered from supershedder cattle. Genome Announc. 3:e01569-14. doi: 10.1128/genomeA.01569-14

Kossow, A., Zhang, W., Bielaszewska, M., Rhode, S., Hansen, K., Fruth, A., et al. (2016). Molecular characterization of human atypical sorbitol-fermenting enteropathogenic Escherichia coli $\mathrm{O} 157$ reveals high diversity. J. Clin. Microbiol. 54, 1357-1363. doi: 10.1128/JCM.02897-15

Larsen, M. V., Cosentino, S., Rasmussen, S., Friis, C., Hasman, H., Marvig, R. L., et al. (2012). Multilocus sequence typing of total-genome-sequenced bacteria. J. Clin. Microbiol. 50, 1355-1361. doi: 10.1128/JCM.06094-11

Medina, A., Horcajo, P., Jurado, S., De la Fuente, R., Ruiz-Santa-Quiteria, J. A., Domínguez-Bernal, G., et al. (2011). Phenotypic and genotypic characterization of antimicrobial resistance in enterohemorrhagic Escherichia coli and atypical enteropathogenic E. coli strains from ruminants. J. Vet. Diagn. Invest. 23, 91-95. doi: 10.1177/104063871102300114

Nataro, J. P., and Kaper, J. B. (1998). Diarrheagenic i. Clin. Microbiol. Rev. 11, $142-201$.

Oswald, E., Schmidt, H., Morabito, S., Karch, H., Marches, O., and Caprioli, A. (2000). Typing of intimin genes in human and animal enterohemorrhagic and enteropathogenic Escherichia coli: characterization of a new intimin variant. Infect. Immun. 68, 64-71. doi: 10.1128/IAI.68.1.64-71.2000

Park, J. H., Oh, S. S., Oh, K. H., Shin, J., Jang, E. J., Jun, B. Y., et al. (2014). Diarrheal outbreak caused by atypical enteropathogenic Escherichia coli O157:H45 in South Korea. Foodborne Pathog. Dis. 11, 775-781. doi: 10.1089/fpd.2014. 1754
Perna, N. T., Mayhew, G. F., Pósfai, G., Elliott, S., Donnenberg, M. S., Kaper, J. B., et al. (1998). Molecular evolution of a pathogenicity island from enterohemorrhagic Escherichia coli O157:H7. Infect. Immun. 66, 3810-3817.

Punina, N. V., Makridakis, N. M., Remnev, M. A., and Topunov, A. F. (2015). Whole-genome sequencing targets drug-resistant bacterial infections. Hum. Genomics 9, 19. doi: 10.1186/s40246-015-0037-z

Reid, S. D., Herbelin, C. J., Bumbaugh, A. C., Selander, R. K., and Whittam, T. S. (2000). Parallel evolution of virulence in pathogenic Escherichia coli. Nature 406, 64-67. doi: 10.1038/35017546

Sanjar, F., Rusconi, B., Hazen, T. H., Koenig, S. S., Mammel, M. K., Feng, P. C., et al. (2015). Characterization of the pathogenome and phylogenomic classification of enteropathogenic Escherichia coli of the O157:non-H7 serotypes. Pathog. Dis. 73:ftv033. doi: 10.1093/femspd/ftv033

Scaletsky, I. C., Souza, T. B., Aranda, K. R., and Okeke, I. N. (2010). Genetic elements associated with antimicrobial resistance in enteropathogenic Escherichia coli (EPEC) from Brazil. BMC Microbiol. 10:25. doi: 10.1186/14712180-10-25

Senerwa, D., Mutanda, L. N., Gathuma, J. M., and Olsvik, O. (1991). Antimicrobial resistance of enteropathogenic Escherichia coli strains from a nosocomial outbreak in Kenya. APMIS 99, 728-734. doi: 10.1111/j.1699. 0463.1991.tb01251.x

Torres, A. G., Zhou, X., and Kaper, J. B. (2005). Adherence of diarrheagenic Escherichia coli strains to epithelial cells. Infect. Immun. 73, 18-29. doi: 10.1128/IAI.73.1.18-29.2005

Trabulsi, L. R., Keller, R., and Tardelli Gomes, T. A. (2002). Typical and atypical enteropathogenic Escherichia coli. Emerg. Infect. Dis. 8, 508-513 doi: 10.3201/eid0805.010385

Zankari, E., Hasman, H., Cosentino, S., Vestergaard, M., Rasmussen, S., Lund, O., et al. (2012). Identification of acquired antimicrobial resistance genes. $J$. Antimicrob. Chemother. 67, 2640-2644. doi: 10.1093/jac/dks261

Zhang, W. L., Köhler, B., Oswald, E., Beutin, L., Karch, H., Morabito, S., et al. (2002). Genetic diversity of intimin genes of attaching and effacing Escherichia coli strains. J. Clin. Microbiol. 40, 4486-4492. doi: 10.1128/JCM.40.12.44864492.2002

Zhou, K., Ferdous, M., de Boer, R. F., Kooistra-Smid, A. M., Grundmann, H., Friedrich, A. W., et al. (2015). The mosaic genome structure and phylogeny of Shiga toxin-producing Escherichia coli O104:H4 is driven by short-term adaptation. Clin. Microbiol. Infect. 21, 468.e7-468.18. doi: 10.1016/j.cmi.2014.12.009

Zhou, Z., Li, X., Liu, B., Beutin, L., Xu, J., Ren, Y., et al. (2010). Derivation of Escherichia coli O157:H7 from its O55:H7 precursor. PLoS ONE 5:e8700. doi: 10.1371/journal.pone.0008700

Conflict of Interest Statement: The authors declare that the research was conducted in the absence of any commercial or financial relationships that could be construed as a potential conflict of interest.

Copyright (C) 2016 Ferdous, Kooistra-Smid, Zhou, Rossen and Friedrich. This is an open-access article distributed under the terms of the Creative Commons Attribution License (CC BY). The use, distribution or reproduction in other forums is permitted, provided the original author(s) or licensor are credited and that the original publication in this journal is cited, in accordance with accepted academic practice. No use, distribution or reproduction is permitted which does not comply with these terms. 